\title{
Young people, the internet and mental health
}

\author{
D. Chambers ${ }^{1, *}$, K. Cairns ${ }^{2}$ and L. Ivancic ${ }^{2}$ \\ ${ }^{1}$ ReachOut Ireland, Dublin, Ireland \\ ${ }^{2}$ ReachOut Australia, Pyrmont, NSW, Australia
}

\begin{abstract}
The original research by Mullen et al. in this issue is a welcome contribution to the increasingly important research area concerned with mental health and internet use. There is a persistent and growing tension between harmful online content and the potential to support vulnerable people online. Although current research has established both negative and positive influences of the internet on mental health, a policy framework to guide the development of online mental health resources is lacking. Based on 20 years of online service provision (in Australia originally and now in Ireland), ReachOut.com has gained important insights into young people's online behaviour and help-seeking preferences. Given that young people have expressed concerns about the impact of the internet and social media on their mental health, yet they will go online for support, there is a clear need for leadership and the resourcing of quality assured, engaging online mental health supports. Such an approach will be the most effective way of mitigating the threat posed by harmful online content.
\end{abstract}

Received 21 September 2017; Revised 25 October 2017; Accepted 27 October 2017; First published online 7 December 2017

Key words: Suicide, the internet, youth mental health.

\section{Introduction: balancing risk and opportunity}

At the intersection of public discourse on the internet and the issue of youth mental health there is a tension between the potential harmful consequences of internet use and the benefits that scalable, evidence-based online resources can achieve. Moreover, the online environment is constantly evolving whereby it is becoming ever easier for users to generate and access text, image or video-based online content on anything and everything of human interest. There is no time lag in the generation of this content, everything is instantaneous. Reflecting this evolution, the relationship between mental health and the internet is complex.

The negative aspects of internet use amongst young people and the inherent risks associated with the online environment have been widely discussed in both academia and the popular media. Cross-sectional, international research such as that conducted by EU Kids Online has provided important insights into risky internet use, especially with the proliferation of mobile phone access (Stald et al. 2014), while high profile cases of cyberbullying and the live broadcast of suicide deaths online have been extensively reported in mainstream media. Further attention has been drawn by the fictional depiction of suicide in drama series broadcast via online streaming sites such as Netflix which, to date, have not adhered to media guidelines adopted by more traditional broadcasters (such as prior warnings around triggering content or the provision of support service details for

* Address for correspondence: D. Chambers, ReachOut Ireland, 32 South William Street, Dublin 2, Ireland.

(Email: chambers.derek@gmail.com) anyone affected by graphic suicide methods). The tendency for people to view this content alone on smartphones and laptops means that any potential for social connection or support for vulnerable viewers is lost.

While this sense of risk and anxiety tends to pervade, there is a need to better understand the dynamics involved in the relationship between individual mental health and internet use. Also, to balance the risk discourse dominant in this area, the benefits of internet use and the potential to develop dedicated online mental health resources warrants increased research interest. Against this backdrop, the paper by Mullen et al. in this issue is important. Apart from elucidating differences in the nature of internet use among groups from across the mental health continuum, their research shines a light on young people's internet use more generally. The prevalence of cyberbullying reported and the engagement with harmful pro-anorexia and suicide sites discussed by Mullen et al. underlines some of the challenges posed by a medium that is characterised by a unique crossgenerational gap in knowledge, whereby adults may be expected to provide guidance and support while being unfamiliar with many online experiences. Among the instructive findings Mullen et al. report, the research highlights the value of parent-child communication in promoting safer internet use as a pattern of safer use was found to be associated with greater parental knowledge of young people's internet activity. It is important that parents and other adults are supported to develop their digital literacy, so that they can engage in meaningful and constructive conversations around online behaviour with the young people in their lives. 


\section{Suicide and the online environment}

While Mullen et al. rightly point out the dearth of research regarding internet use among young people with mental ill health, there has been considerable recent research published on the internet and suicide which is highly relevant to youth mental health more broadly. This research has ranged from exploration of the online environment itself (e.g. Biddle et al. 2016) to a systematic review of the relationship between internet use and suicidal behaviour (Marchant et al. 2017). The study by Biddle et al. (2016) followed on from a similar study by the same researchers (Biddle et al. 2008), which involved online searches for suicide-related information to determine the type of content that is likely to be accessed by suicidal internet users. The fieldwork (searches) for these respective publications took place in 2014 and 2007. An important difference in relation to online content retrieved was the proliferation of usergenerated content on social media, blogs and personal websites, representing a more diffuse online environment in 2014. Worryingly, there was a decrease between 2007 and 2014 in the percentage of prevention or support sites - from $13 \%$ to $6.5 \%$ - that were returned by the searches. Among the recommendations from this research, it was highlighted that 'working with internet service providers and search engines is likely to be a key strategy for tipping the balance of the information that is accessed' (towards support sites).

Biddle et al.'s research provides an important overview of the online environment but it does not help us to better understand user engagement as it relates to mental health, help-seeking or suicidal behaviour. A recent systematic review explored various aspects of the relationship between internet use and suicidal behaviour in young people (Marchant et al. 2017). Examining literature published from 2011 to 2014, Marchant et al.'s review included 46 independent studies which reported a range of influences on suicidal behaviour. In total, 18 studies reported negative influences, 11 were positive and influences were mixed for 17 studies. The authors of this review concluded that, 'there is a significant potential for harm to result from online behaviour in relation to self-harm and suicidal behaviour (normalisation, triggering and competition between users, a source of contagion and harmful information for vulnerable individuals), but also the potential to exploit its benefits (a sense of community, crisis support and reduction of social isolation)'.

\section{The experience of delivering ReachOut.com}

With regards to the potential benefits of the internet in supporting people, the ReachOut.com service has been providing supportive online content on mental health to young people since the 1990s. When it launched in
Australia in 1998, ReachOut.com became the world's first online mental health service, whilst ReachOut was launched in Ireland in late 2009. ReachOut.com focuses on the provision of engaging online content on a broad range of mental health-related issues, including usergenerated content in the form of real stories and videos. An important element of the service ethos involves the provision of practical self-help strategies that can be adopted in 'the here and now' in addition to signposting distressed users to crisis support services.

From 2011 to 2015, ReachOut Ireland conducted annual user surveys to evaluate the service. These surveys were published on ReachOut.com and routinely followed the methodology set out in the first report (see Chambers \& Murphy, 2011). These surveys were completed by an annual average of 211 selfselecting respondents (total $n=1053$, ranging from 98 to 283 in 2015 and 2012, respectively). Of the total 5 -year sample: over $60 \%$ of participants reported moderate or severe levels of psychological distress; 'online' was the most frequently selected likely source of support (64\%); and, the least often selected source of support was a 'phone helpline' (14\%). Responding to an open-ended question about barriers to support, the common theme of fear was articulated by one of the respondents who said 'I feel like if I tell someone, it becomes real and a part of me. If I deal with it on my own it's just a blip'. Overcoming this fear of disclosure is a key way in which online resources can facilitate a young person's help-seeking journey, as outlined in Rickwood et al.'s (2005) model of help-seeking, which describes the first step in getting appropriate support as recognising and understanding more about the issue that is troubling you. Online resources can facilitate this step in a free-to-access, round the clock, anonymous and confidential manner.

In 2014, ReachOut Australia initiated a longitudinal study (Metcalf et al. 2015) to better understand the characteristics of their users, their motivations for visiting, and how the site helps them. This study involved a rolling sample of 2000 users of the Australian website aged between 16 and 25 years. In total, $17 \%$ of participants were classified as being at high risk of suicide, whilst a further $23 \%$ were classified as having recent thoughts of suicide, as assessed by the Suicidal Ideation Questionnaire, and based on work by Furlong et al. (personal communication, 21 July 2017). The vast majority of participants identified their primary motivation for visiting ReachOut as being to access information and support for depression (including $43 \%$ of those with recent thoughts of suicide and $48 \%$ of those considered to be at high risk) or anxiety (33\% of those with recent thoughts and 30\% of those at high risk). A help-negation effect (Ivancic \& Buhagiar, 2017) was also found, whereby as suicidal 
ideation increased, participants were less likely to report that they would seek help from either social support or face-to-face, phone and/or online support, and were more likely to say they would seek help from no-one or no-where.

Whilst this is concerning, it is encouraging that the majority of the participants who were experiencing suicidal ideation reported that ReachOut was helping them. For example, $71 \%$ of those with recent thoughts of suicide agreed or strongly agreed with the statement 'ReachOut helps me understand my experience' and $86.9 \%$ of the same group agreed or strongly agreed with the statement 'ReachOut is available and accessible in ways that are convenient for me'. This longitudinal study confirms that young people are explicitly seeking out information about suicide online when they do not feel comfortable going elsewhere, and suggests that online services like ReachOut.com have an important role to play in providing immediate relief to young people in high distress, and in facilitating their access to informal (e.g. family members, friends) and/or formal (e.g. mental health professional) sources of support.

\section{Conclusion: capturing the voice of young people}

Apart from annual user surveys in Ireland and the longitudinal research with users in Australia, ReachOut have also conducted community surveys to better understand our users and audience. In early 2017, in Ireland, an online survey of teenagers was conducted in partnership with a national, daily broadsheet newspaper, which attracted over 2500 respondents in just 3 days (Chambers et al. 2017). The survey included structured questions about help-seeking preferences and an open question asking for general comments in relation to youth mental health today. This open-ended question was answered by 1143 teenagers, and their responses were coded utilising the thematic analysis method described by Braun \& Clarke (2006), whereby themes were data-driven, rather than theory-driven, in order to give voice to the young respondents. Of the eight themes identified, one particularly relevant to this editorial was 'social media' and its relationship to teenage mental health.

There were frequent, strong and polarised views on social media and mental health expressed in the survey. A prominent view that was expressed centred around the weight of expectation and pressure that social media places on teenagers. While the majority of those who mentioned social media were negative about the issue, there were other more positive views. Among the most positive endorsements of social media, mobile phones and the role they play in a teenager's life was the view that 'we hear all these stories about phones and social media being bad for our health but the truth is if I didn't have a phone I wouldn't be able to cope with life, it distracts us from a lot of our problems and the fact is that my generation of teens have grown up using smartphones, there's no going back now'.

There is no going back now. Effective strategies to promote positive and healthy use of the internet must be pursued in order to ensure young people have access to reliable, endorsed and trustworthy online services that provide information on mental health issues and support options. The provision of these types of services will respond to well-established help-seeking preferences and behaviours amongst young people, and can go a long way to mitigating the negative influences of harmful online content.

\section{Financial Support}

This article received no specific grant from any funding agency, commercial or not-for-profit sectors.

\section{Conflicts of Interest}

The authors are employed by ReachOut Ireland (D.C.) and ReachOut Australia (K.C. and L.I.), the organisations which operate ReachOut.com, an online service referenced throughout the editorial.

\section{Ethical Standards}

The authors assert that all procedures contributing to this work comply with the ethical standards of the relevant national and institutional committee on human experimentation with the Helsinki Declaration of 1975 , as revised in 2008 .

\section{References}

Biddle L, Derges J, Mars B, Heron J, Donovan J, Potokar J, Piper M, Wyllie C, Gunnell D (2016). Suicide and the internet: changes in the accessibility of suicide-related information between 2007 and 2014. Journal of Affective Disorders 190, 370-375.

Biddle L, Donovan J, Hawton K, Kapur N, Gunnell D (2008). Suicide and the internet. British Medical Journal 336, 800-802.

Braun V, Clarke V (2006). Using thematic analysis in psychology. Qualitative Research in Psychology 3, 77-101.

Chambers D, Murphy F (2011). Learning to Reach Out, Young People, Mental Health Literacy and the Internet. Inspire Ireland Foundation: Dublin, Ireland.

Chambers D, Ryan F, Doolan R, Kavanagh N, Healy C (2017). What's Wrecking Your Head? - A Report on Teenage Mental Health. ReachOut Ireland: Dublin, Ireland.

Ivancic L, Buhagiar K (2017). Understanding how an online prevention and early intervention service can effectively engage and support visitors at risk of suicide. Paper presented at the National Suicide Prevention Conference 2017, Brisbane, QLD. 
Marchant A, Hawton K, Stewart A, Montgomery P, Singaravelu V, Lloyd K, Purdy N, Daine K, John A (2017). A systematic review of the relationship between internet use, self-harm and suicidal behaviour in young people: the good, the bad and the unknown. PLoS ONE 12, e0181722.

Metcalf A, Ivancic L, Blake V, Buhagiar K, Wilson C, Kauer S, Sanci L (2015). ReachOut.com Cohort Study: a prospective follow-up study examining the role of online initiatives in promoting mental health literacy and early help-seeking. Paper presented at the 3rd International Conference on Youth Mental Health 2017, Brisbane, QLD. Rickwood D, Deane FP, Wilson CJ, Ciarrochi J (2005).

Young people's help-seeking for mental health problems. Australian e-Journal for the Advancement of Mental Health 4, 1-34. Stald G, Green L, Barbovski M, Haddon L, Mascheroni G, Ságvári B, Scifo B, Tsaliki L (2014). Online on the Mobile: Internet Use on Smartphones and Associated Risks Among Youth in Europe. EU Kids Online: London, UK. 